\title{
Quantification of peripapillary vessel density in non-arteritic anterior ischemic optic neuropathy patients with optical coherence tomography angiography
}

\author{
Yufang Su ${ }^{1 \#}$, Song Zhang ${ }^{2 \#}$, Guisen Zhang ${ }^{3 \#}$, Yingru Liu ${ }^{1}$, Zhiguo Du $^{1}$, Daming $\mathrm{Li}^{4}$, Lei Liu ${ }^{5,6,7}$ \\ ${ }^{1}$ Department of Glaucoma, Baotou Chaoju Eye Hospital, Baotou, China; ${ }^{2}$ China Medical University, Shenyang, China; ${ }^{3}$ Department of Retina, \\ Inner Mongolia Chaoju Eye Hospital, Hohhot, China; ${ }^{4}$ Department of Glaucoma, Dalate Qi Chaoju Eye Hospital, Erdos, China; ${ }^{5}$ Department \\ of Ophthalmology, The First Affiliated Hospital of China Medical University, Shenyang, China; ${ }^{6}$ Guangdong Eye Institute, Department of \\ Ophthalmology, Guangdong Provincial People's Hospital, Guangdong Academy of Medical Sciences, Guangzhou, China; ${ }^{7}$ School of Medicine, \\ South China University of Technology, Guangzhou, China
}

Contributions: (I) Conception and design: Y Su, L Liu; (II) Administrative support: L Liu; (III) Provision of study materials or patients: Y Su, Y Liu, Z Du; (IV) Collection and assembly of data: Y Su, Y Liu, Z Du; (V) Data analysis and interpretation: S Zhang, L Liu; (VI) Manuscript writing: All authors; (VII) Final approval of manuscript: All authors.

"These authors contributed equally to this work.

Correspondence to: Daming Li. Department of Glaucoma, Dalate Qi Chaoju Eye Hospital, Notarization Building, Desheng Street, Shulinzhao Town, Dalate Banner, Erdos 014300, China. Email: 1178520726@qq.com; Lei Liu. Department of Ophthalmology, The First Affiliated Hospital of China Medical University, No. 155 Nanjing North Street, Heping District, Shenyang 110122, China; Guangdong Eye Institute, Department of Ophthalmology, Guangdong Provincial People's Hospital, Guangdong Academy of Medical Sciences; School of Medicine, South China University of Technology, No. 106, Zhongshan 2nd Road, Yuexiu District, Guangzhou 510120, China. Email: liuleijiao@163.com.

Background: Quantitative assessments based on optical coherence tomographic angiography (OCTA) may have potential promising value in the early detection of non-arteritic anterior ischemic optic neuropathy (NA-AION), but there is limited information on the ability of OCTA to distinguish eyes with NA-AION. This study was conducted to evaluate the ability of measurements of peripapillary perfusion using OCTA to distinguish healthy eyes from eyes with NA-AION.

Methods: In this retrospective case-control study, newly diagnosed NA-AION patients and healthy controls matched at a ratio of 1:3 by gender and age ( \pm 5 years) were enrolled from 1 September 2020 to 30 June 2021. Peripapillary vessel density (pVD) was examined based on the area of vessels by means of a 4.5 mm OCTA scan. In addition, peripapillary retinal nerve fiber layer (pRNFL) thickness was obtained from structural optical coherence tomography (OCT), as was the area under the receiver operating characteristic (ROC) curve (AUC).

Results: A total of 29 eyes from 28 cases with NA-AION and 99 healthy eyes from 68 participants were imaged. All participants were Chinese. The NA-AION group showed a significant reduction of the pVD $(\mathrm{P}<0.001)$, while all subregions of $\mathrm{pRNFL}$ thickness were prominent in all 8 quadrants $(\mathrm{P}>0.05)$. The $\mathrm{pVD}$ of the optic disc in the superior temporal (ST) region showed better diagnostic accuracy (AUC $=0.86$ ) in discriminating the NA-AION group from healthy controls. After adjusting for confounders, ST was independently associated with the presence of NA-AION [odds ratio (OR) $=0.971,95 \%$ confidence interval (CI): 0.943-0.990, P=0.048].

Conclusions: Decreased pVD was detected by non-invasive measurements of OCTA in the eyes of NAAION patients. This finding may reveal an association between the ST region and the presence of NAAION. The pVD may have potential diagnostic ability and may serve as an additional biomarker in the management of the disease. 


\begin{abstract}
Keywords: Non-arteritic anterior ischemic optic neuropathy (NA-AION); optical coherence tomography (OCT); optical coherence tomography angiography (OCTA); peripapillary vessel density (pVD)
\end{abstract}

Submitted Aug 12, 2021. Accepted for publication Oct 29, 2021.

doi: $10.21037 /$ qims-21-800

View this article at: https://dx.doi.org/10.21037/qims-21-800

\section{Introduction}

Non-arteritic anterior ischemic optic neuropathy (NAAION) is the most common type of optic neuropathy and one of the most prevalent and visually crippling diseases in adults over 50 years of age (1). Its pathogenesis involves the transient hypoperfusion or non-perfusion of the optic disc vascular bed, which is caused by the temporary decrease in the perfusion pressure of the posterior ciliary artery supplying the lamina area and posterior lamina area of the optic papilla, leading to the apoptosis of retinal ganglion cells (RGCs) or degeneration of the optic nerve $(2,3)$. The occlusion of the posterior ciliary artery, which is generally considered to be only related to the occurrence of inflammatory anterior ischemic arteries, is also related to the occurrence of neuropathy (4).

Clinically, NA-AION usually presents as sudden, painless monocular vision loss, accompanied by characteristic visual field defects, opposing pupil conduction block, and optic disc edema, with or without optic disc peri-optical nerve fibers thickness, and may result in optic nerve damage, seriously impaired vision, or blindness (1). Early detection is required for management of the disease (5). However, there is still no gold standard for the diagnosis of NAAION, and a comprehensive diagnosis based on typical clinical symptoms, fundus manifestations, visual field, and fundus fluorescein angiography (FFA) changes is required in the clinical setting. Optical coherence tomography angiography (OCTA), with no injectable dye or enhanced capillary visualization properties, is a new vascular imaging technology that can quickly and non-invasively display the vascular morphology and vessel density around the optic disc and the macular area (6-8). It can display qualitative analysis of the retina and choroidal vasculature disorders as well as optic neuropathy. Previously, few studies have described changes of the microvasculature in NA-AION using OCTA (9-12). However, it is worth noting that there is limited information on the ability of OCTA to distinguish eyes with NA-AION.

This article aims to evaluate the measurement ability of peripapillary vessel density (pVD) using OCTA to distinguish healthy eyes from eyes with NA-AION, so as to provide a basis for the rapid diagnosis of the disease. We present the following article in accordance with the STROBE reporting checklist (available at https://dx.doi. org/10.21037/qims-21-800).

\section{Methods}

\section{Subjects}

This was a retrospective case-control study, including newly diagnosed NA-AION patients and healthy controls who were selected from the general population, individually matched at a ratio of $1: 3$ by gender and age ( \pm 5 years), and examined at the Ophthalmology Unit, Baotou Chaoju Eye Hospital, Baotou, Inner Mongolia Autonomous Region, China, from 1 September 2020 to 30 June 2021. The study was conducted in accordance with the Declaration of Helsinki (as revised in 2013). The study was approved by the Medical Ethics Committee of the Baotou Chaoju Eye Hospital and informed consent was provided by all participants.

The following criteria were used to confirm NA-AION diagnosis: (I) sudden and painless vision loss; (II) optic disc edema, or a small amount of hemorrhage around the optic disc; (III) quadrant defect of the visual field, with horizontal or vertical hemianopia; (IV) FFA showing early stage partial or full disc filling delay, and intense fluorescence or fluorescein leakage at the late stage; $(\mathrm{V})$ the absence of neurological diseases by magnetic resonance imaging (MRI).

The exclusion criteria were as follows: (I) severe refractive interstitial turbidity or severe fundus disease affecting the OCTA examination; (II) inflammatory, genetic, traumatic, and other causes of optic nerve disease; (III) history of intraocular surgery, laser, and medication in the past 6 months.

The time span for considering the patients as "newly" diagnosed was 10 days. All NA-AION participants underwent a complete eye examination, consisting of best corrected visual acuity (BCVA), slit-lamp evaluation, intraocular pressure (IOP), dilated fundus evaluation, 


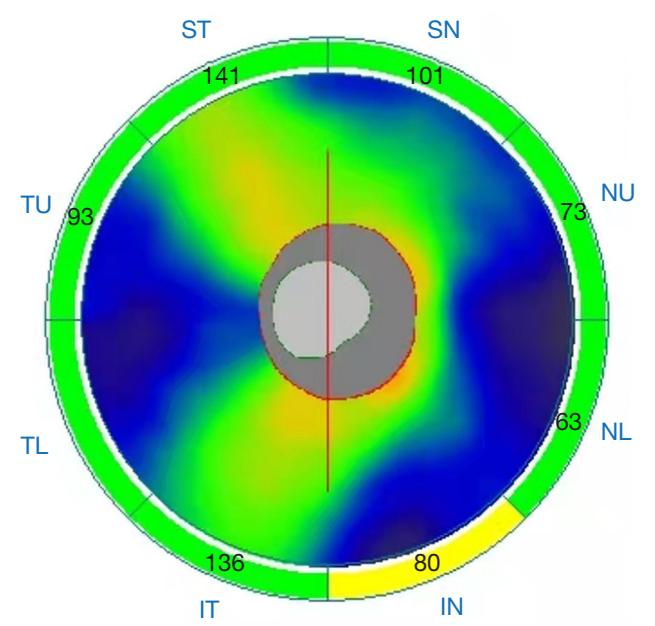

Figure 1 Diagram of the procedure for $\mathrm{ONH}$ measurement by OCT. ONH, optic nerve head; OCT, optical coherence tomography; pTU, peripapillary temporal upper; pST, peripapillary superior temporal; pSN, peripapillary superior nasal; pNU, peripapillary nasal upper; pNL, peripapillary nasal lower; pIN, peripapillary inferior nasal; pIT, peripapillary inferior temporal; pTL, peripapillary temporal lower.

FFA (Heidelberg Engineering, Heidelberg, Germany), automated visual field examination (24-2 Swedish Interactive Threshold Algorithm; Humphrey Field Analyser II; Carl Zeiss Meditec, Inc., Dublin, CA, USA), and flash visual evoked potential (FVEP) test (UTAS-E3000LKC, Multifocal Visual Diagnostic Test System, IKC Technologies, Gaithersburg, MD, USA). Refraction was measured with an autorefractometer and described as spherical equivalent (spherical dioptric power plus half of the cylindrical dioptric power). Medical conditions, including diabetes mellitus, systemic hypertension, hypercholesterolemia, and atherosclerotic carotid artery plaque were recorded. All controls underwent a complete eye examination with all the items above except FFA.

Two experienced ophthalmologists, who independently interpreted the optic changes, were blinded to the findings of conventional ophthalmoscopy.

\section{Radial peripapillary capillary measurement}

Structural OCT images with quantitative measures in all regions of peripapillary retinal nerve fiber layer (pRNFL) thickness (peripapillary temporal upper, pTU; peripapillary superior temporal, pST; peripapillary superior nasal,

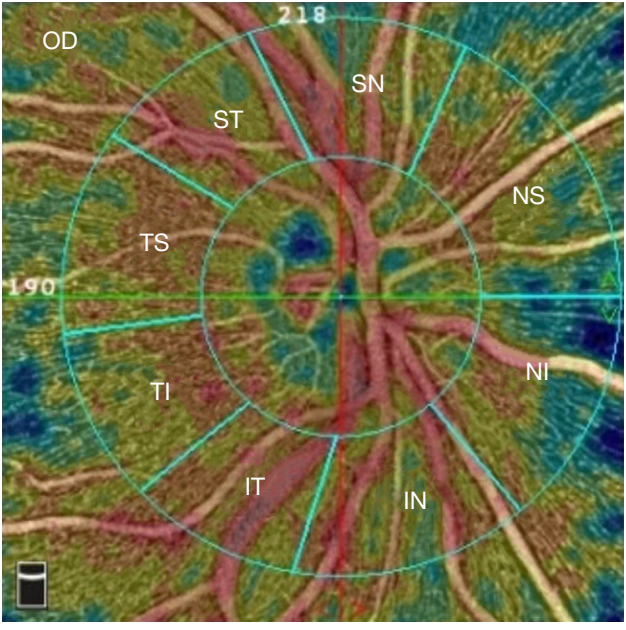

Figure 2 Peripapillary optic disc blood flow via OCTA. OCTA, optical coherence tomography angiography; NS, nasal superior; NI nasal inferior; IN inferior nasal; IT, inferior temporal; TI, temporal inferior; TS, temporal superior; ST, superior temporal; $\mathrm{SN}$, superior nasal.

pSN; peripapillary nasal upper, pNU; peripapillary nasal lower, pNL; peripapillary inferior nasal, pIN; peripapillary inferior temporal, pIT; peripapillary temporal lower, pTL, Figure 1) were automatically acquired using AngioVue OCTA system version 2017.1 (Optovue Inc., Fremont, CA, USA). Subsequently, the AngioVue OCTA system was set at Angio-Disc mode, and a $4.5 \mathrm{~mm} \times 4.5 \mathrm{~mm}$ scan centered on the optic nerve head $(\mathrm{ONH})$ was obtained for each eye. The instrument has an A-scan rate of 70,000 per second, using a light source centered on $840 \mathrm{~nm}$. We included only high-quality images — defined by scans with scan quality $>7 / 10$ (and without artifacts). The software automatically fits a circle with a diameter of $2.0 \mathrm{~mm}$, centered on the $\mathrm{ONH}$, which extends from the $\mathrm{ONH}$ to the peripapillary area. All images were re-evaluated by an expert ophthalmologist (Y.F.S) and manually corrected if necessary. The peripapillary area was divided into 8 subregions automatically according to the GarwayHeath method (13): nasal superior (NS), nasal inferior (NI), inferior nasal (IN), inferior temporal (IT), temporal inferior (TI), temporal superior (TS), superior temporal (ST), and superior nasal (SN) (Figure 2). Vessel densities of each sector of the peripapillary area were generated by the software automatically. 

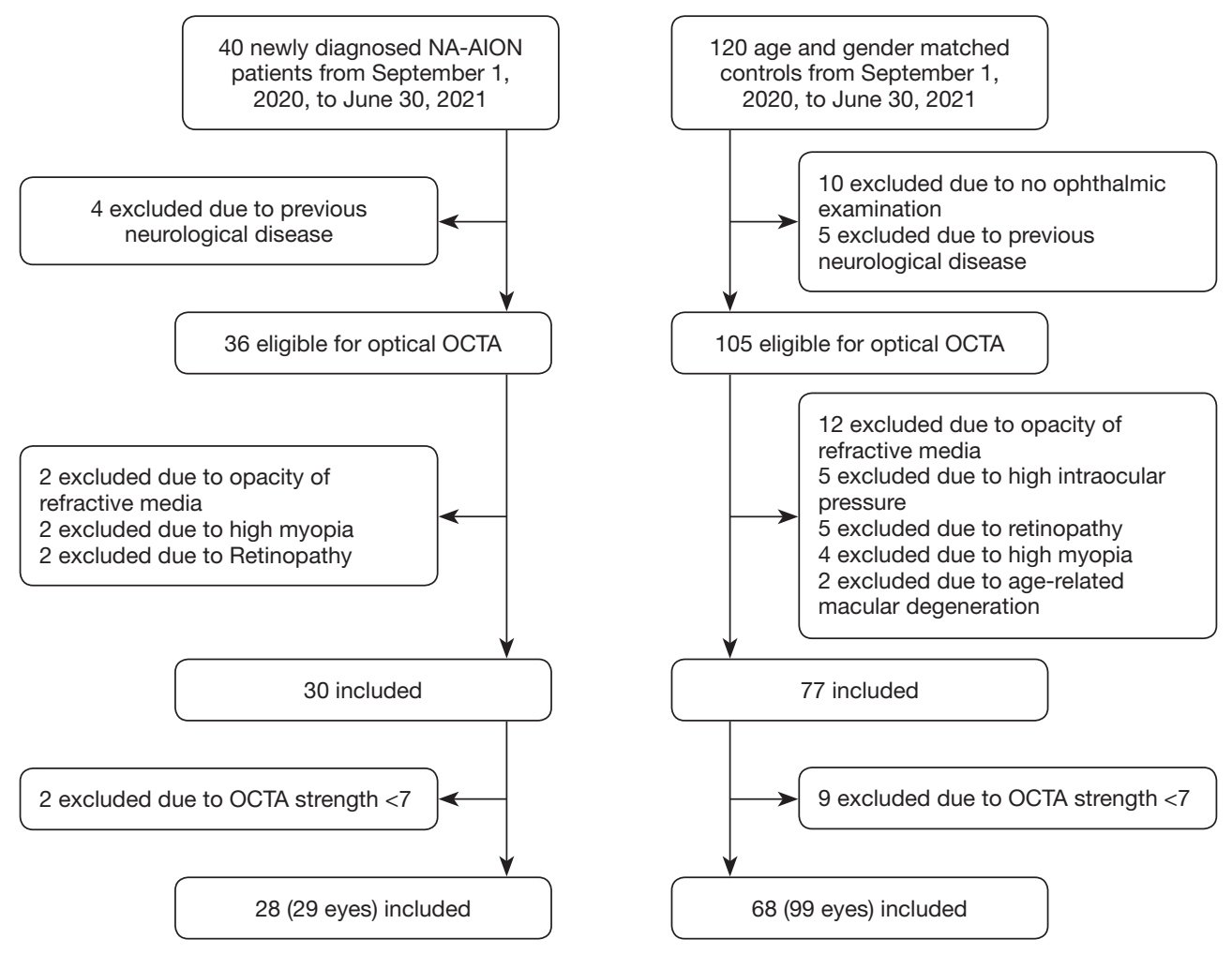

Figure 3 Flow diagram of the study population. OCTA, coherence tomography angiography.

\section{Statistical analysis}

Data was described as mean \pm standard deviation $(\mathrm{SD})$ or median and interquartile range (IQR) depending on its distribution. The Kolmogorov-Smirnov test was used to assess variable distribution. The paired two-sample Student's $t$-test or Wilcoxon test was used to assess statistical differences between groups. The ability of quantitative measures of pRNFL thickness and vessel densities of each sector of peripapillary area indexes to predict NA-AION was determined using receiver operating characteristic (ROC) curve analysis. The optimal cutoff for sensitivity and specificity was calculated by maximizing the Youden's index. The multivariate logistic regression model, including all covariates found to be significantly associated with NAAION in the univariate analysis, was used. Odds ratio (OR) and $95 \%$ confidence interval (CI) were determined. All statistical analysis was performed using IBM SPSS Statistics (V.20.0 (International Business Machine Corporation, Armonk, NY, USA). A P value $<0.05$ was considered statistically significant.

\section{Results}

A total of 29 eyes from 28 patients with newly diagnosed NA-AION (mean age $57.97 \pm 7.26$ years) and 99 eyes from 68 controls (mean age $57.86 \pm 7.50$ years) were included in this study (Figure 3). All cases and controls were sampled together from a hospital based on the presence of a record regardless of the presence of NA-AION. There were no significant differences in age, gender, medical history (presence of diabetes, systemic hypertension, and atherosclerotic carotid artery plaque), spherical equivalent, and latency of $\mathrm{P}_{100}$ wave in FVEP between the 2 groups $(\mathrm{P}>0.05)$. The NA-AION group had lower BCVA, mean deviation (MD), and pattern standard deviation (PSD) but higher visual field index (VFI) than the control group (Table 1, $\mathrm{P}<0.001$ ).

The mean measurements of all the pRNFL parameters investigated were not significantly different in the 2 groups (Table 2, $\mathrm{P}>0.05$ ). However, there was a significant difference pertaining to the 8 subregions of pVD (Table 2, $\mathrm{P}<0.001)$. 
Table 1 Demographic and clinical characteristics of study participants

\begin{tabular}{|c|c|c|c|}
\hline Variable & $\begin{array}{c}\text { NA-AION } \\
(n=29)\end{array}$ & $\begin{array}{c}\text { Control } \\
(n=99)\end{array}$ & $P$ value \\
\hline Age (years) & $57.97 \pm 7.26$ & $57.86 \pm 7.50$ & 0.946 \\
\hline Male, n (\%) & $13(44.83)$ & $50(50.51)$ & 0.591 \\
\hline With diabetes, n (\%) & $4(13.79)$ & $9(9.09)$ & 0.461 \\
\hline With hypertension, n (\%) & $17(58.62)$ & $41(41.41)$ & 0.102 \\
\hline $\begin{array}{l}\text { With hypercholesterolemia, n } \\
(\%)\end{array}$ & $2(6.90)$ & $6(6.06)$ & 0.870 \\
\hline $\begin{array}{l}\text { With atherosclerotic carotid } \\
\text { artery plaque, } \mathrm{n}(\%)\end{array}$ & $6(20.69)$ & 9 (9.09) & 0.088 \\
\hline BCVA & $0.66 \pm 0.35$ & $0.94 \pm 0.16$ & $<0.001$ \\
\hline Spherical equivalent (D) & $-0.51 \pm 1.40$ & $-0.18 \pm 1.49$ & 0.282 \\
\hline \multicolumn{4}{|l|}{ Visual field } \\
\hline VFI \% & $52.41 \pm 25.57$ & $93.13 \pm 16.85$ & $<0.001$ \\
\hline $\mathrm{MD}(\mathrm{dB})$ & $-15.70 \pm 7.85$ & $-3.51 \pm 5.00$ & $<0.001$ \\
\hline PSD (dB) & $10.20 \pm 4.24$ & $2.94 \pm 2.04$ & $<0.001$ \\
\hline \multicolumn{4}{|l|}{ FVEP } \\
\hline $\mathrm{P}_{100}$ latency & $64.41 \pm 55.59$ & $88.45 \pm 38.92$ & 0.627 \\
\hline $\mathrm{P}_{100}$ amplitude & $7.57 \pm 7.90$ & $13.62 \pm 7.97$ & $<0.001$ \\
\hline
\end{tabular}

BCVA, best corrected visual acuity; FEVP, flash visual evoked potential; MD, mean deviation; NA-AION, non-arteritic anterior ischemic optic neuropathy; PSD, pattern standard deviation; $\mathrm{VFI}$, visual field index.

Table 3 shows the ROC curve analysis results for measurements of all the pRNFL parameters and 8 subregion vessel densities of the ONH. The area under the curve (AUC) values showed good specificity for the 8 subregion vessel densities of the ONH, especially ST (AUC $=0.86$ ). Among NA-AION patients, the optimum cutoff of ST vessel density was 48.5 , with $86.2 \%$ sensitivity and $84.8 \%$ specificity.

The adjusted OR of ST for NA-AION patients with adjustment for factors including BCVA, VFI, MD, PSD, and $\mathrm{AP}_{100}$ was 0.97 (95\% CI: 0.94-0.99, $\mathrm{P}=0.048$, Table 4).

\section{Discussion}

NA-AION is the most common cause of acute optic nerve ischemia and the leading cause of acute loss of vision in people over 55 years of age. This condition is thought to result from circulatory failure within the optic disc. Our
Table 2 Characteristics of pRNFL and vessel density among the participants

\begin{tabular}{|c|c|c|c|}
\hline Variable & NA-AION ( $n=29)$ & Control $(n=99)$ & $P$ value \\
\hline \multicolumn{4}{|c|}{ Peripapillary retinal nerve fiber layer } \\
\hline pST & $120.10 \pm 101.25$ & $124.24 \pm 48.01$ & 0.387 \\
\hline $\mathrm{pSN}$ & $100.90 \pm 84.35$ & $103.10 \pm 41.96$ & 0.469 \\
\hline $\mathrm{pNU}$ & $87.00 \pm 73.96$ & $73.84 \pm 29.83$ & 0.134 \\
\hline $\mathrm{pNL}$ & $89.62 \pm 78.23$ & $65.48 \pm 26.82$ & 0.109 \\
\hline $\mathrm{pIN}$ & $115.07 \pm 99.12$ & $103.78 \pm 43.15$ & 0.322 \\
\hline plT & $116.52 \pm 93.65$ & $124.49 \pm 48.73$ & 0.214 \\
\hline pTL & $72.72 \pm 63.24$ & $63.06 \pm 24.18$ & 0.366 \\
\hline pTU & $90.97 \pm 78.83$ & $76.94 \pm 31.24$ & 0.190 \\
\hline \multicolumn{4}{|c|}{ Peripapillary optic disc vessel density } \\
\hline ST & $28.21 \pm 19.82$ & $50.05 \pm 18.60$ & $<0.001$ \\
\hline SN & $27.24 \pm 18.37$ & $46.13 \pm 17.74$ & $<0.001$ \\
\hline NS & $29.07 \pm 19.35$ & $44.34 \pm 16.29$ & $<0.001$ \\
\hline $\mathrm{NI}$ & $30.45 \pm 20.73$ & $43.83 \pm 15.68$ & $<0.001$ \\
\hline IN & $30.83 \pm 21.14$ & $47.66 \pm 17.27$ & $<0.001$ \\
\hline IT & $32.28 \pm 23.47$ & $52.33 \pm 19.09$ & $<0.001$ \\
\hline $\mathrm{TI}$ & $34.00 \pm 23.22$ & $48.28 \pm 17.02$ & $<0.001$ \\
\hline TS & $34.93 \pm 23.17$ & $51.31 \pm 18.26$ & $<0.001$ \\
\hline
\end{tabular}

NA-AION, non-arteritic anterior ischemic optic neuropathy; pTU, peripapillary temporal upper; pST, peripapillary superior temporal; pSN, peripapillary superior nasal; pNU, peripapillary nasal upper; pNL, peripapillary nasal lower; pIN, peripapillary inferior nasal; pIT, peripapillary inferior temporal; pTL, peripapillary temporal lower; NS, nasal superior; NI nasal inferior; IN inferior nasal; IT, inferior temporal; TI, temporal inferior; TS, temporal superior; ST, superior temporal; SN, superior nasal.

results revealed that the difference in pRNFL was not statistically significant between the NA-AION and control groups, while the vessel density was lower than the healthy controls in all quadrants. Furthermore, the pVD in the ST area showed better diagnostic accuracy (AUC $=0.86$ ) in discriminating the NA-AION group from the healthy controls. The pVD in the ST area was independently associated with the presence of NA-AION. These findings may provide additional information by OCTA for the diagnosis of microvascular defects and VD reduction in new cases of NA-AION among adolescents.

Generally, many systemic disorders including 
Table 3 Receiver operating characteristic curves and prognostic accuracy of pRNFL and vessel density in non-arteritic anterior ischemic optic neuropathy

\begin{tabular}{lllllll}
\hline Variable & AUC & $95 \% \mathrm{Cl}$ & P value & Cutoff & Sensitivity (\%) & Specificity (\%) \\
\hline Peripapillary retinal nerve fiber layer & & & & & & \\
pST & 0.55 & $0.38-0.72$ & 0.388 & 162 & 48.3 & 92.9 \\
pSN & 0.54 & $0.38-0.70$ & 0.470 & 135.5 & 48.3 & 86.9 \\
pNU & 0.59 & $0.43-0.76$ & 0.135 & 107.5 & 51.7 & 94.9 \\
pNL & 0.59 & $0.43-0.76$ & 0.110 & 99.5 & 51.7 & 97.0 \\
pIN & 0.56 & $0.39-0.72$ & 0.323 & 148.5 & 44.8 & 93.9 \\
pIT & 0.57 & $0.41-0.74$ & 0.215 & 167.5 & 51.7 & 94.9 \\
pTL & 0.55 & $0.39-0.72$ & 0.367 & 93 & 44.8 & 97.0 \\
PTU & 0.58 & $0.41-0.75$ & 0.191 & 109 & 55.2 & 99.0 \\
Peripapillary vessel density & & & & & 86.2 \\
ST & 0.86 & $0.79-0.93$ & $<0.001$ & 48.5 & 86.2 & 84.8 \\
SN & 0.85 & $0.79-0.92$ & $<0.001$ & 46.5 & 96.6 & 76.8 \\
NS & 0.82 & $0.74-0.90$ & $<0.001$ & 45.5 & 86.2 & 74.7 \\
NI & 0.75 & $0.65-0.85$ & $<0.001$ & 43.5 & 65.5 & 78.8 \\
IN & 0.80 & $0.71-0.90$ & $<0.001$ & 48.5 & 89.7 & 80.8 \\
IT & 0.80 & $0.71-0.89$ & $<0.001$ & 52.5 & 82.8 & 81.8 \\
TI & 0.72 & $0.61-0.84$ & $<0.001$ & 49.5 & 65.5 & 75.8 \\
TS & 0.82 & $0.74-0.90$ & $<0.001$ & 53.5 & 82.8 & 78.8 \\
\hline AUC, a & & & &
\end{tabular}

AUC, area under the receiver operating characteristic curve; $\mathrm{Cl}$, confidence interval; pTU, peripapillary temporal upper; pST, peripapillary superior temporal; pSN, peripapillary superior nasal; pNU, peripapillary nasal upper; pNL, peripapillary nasal lower; pIN, peripapillary inferior nasal; pIT, peripapillary inferior temporal; pTL, peripapillary temporal lower; NS, nasal superior; NI nasal inferior; IN inferior nasal; IT, inferior temporal; TI, temporal inferior; TS, temporal superior; ST, superior temporal; SN, superior nasal.

systemic hypertension $(14,15)$, diabetes mellitus (16-18), hypercholesterolemia $(19,20)$, and atherosclerotic carotid artery plaque (21) have been reported as risk factors for NAAION as these disorders may reduce optic disc perfusion $(22,23)$. In the current matched case-control study, there was no difference in these systemic disorders between the NA-AION and control groups. Although there were significant differences in visual field measurements (MD, PSD, and VFI) and BCVA between optic nerve morphology in NAION and control eyes, these variables were adjusted in the multivariate logistic regression model. Among newly diagnosed NA-AION cases, damage with inflammatory/ exudative reactions on the optic nerve may lead to variable $\mathrm{ONH}$ edema (24). In a previous case-control study, the authors observed that the regions that were useful in the diagnosis of NAION were pST, pSN, pNU, and pIT (25), which was inconsistent with our findings. Optic edema in NA-AION is secondary to the reduction of blood flow to the $\mathrm{ONH}$ by the phenomenon of crowding on a small excavation disc (26). Thus, it is necessary to find biomarkers other than pRNFL to identify NA-AION.

The non-invasive, depth-resolved imaging tool OCTA has been widely used in the assessment of peripapillary and macular capillary changes (27). The spectrum of neuroophthalmological diseases shows consistent changes in the capillaries around the $\mathrm{ONH}$ and the macula (28). Recently, the role of OCTA in the diagnosis and management of optic nerve-related diseases has gradually been recognized (29). A previous case-control study involving 21 eyes with no acute NA-AION and 19 healthy control eyes revealed that the optic disc non-perfusion area percentages in the non-acute phase NA-AION patients detected via OCTA 
Table 4 Multivariate logistic regression models, including all covariates found to be significantly associated with NA-AION in the univariate analysis

\begin{tabular}{lccc}
\hline Variable & OR ${ }^{*}$ & $95 \% \mathrm{Cl}$ & P value \\
\hline \multicolumn{3}{c}{ Peripapillary retinal nerve fiber layer } & \\
pST & 1.01 & $0.99-1.02$ & 0.091 \\
pSN & 1.01 & $1.00-1.03$ & 0.049 \\
pNU & 1.02 & $1.00-1.04$ & 0.054 \\
pNL & 1.02 & $0.99-1.04$ & 0.077 \\
pIN & 1.01 & $0.99-1.02$ & 0.184 \\
pIT & 1.01 & $0.99-1.02$ & 0.392 \\
pTL & 1.01 & $0.99-1.03$ & 0.194 \\
pTU & 1.02 & $1.00-1.04$ & 0.037 \\
Peripapillary optic disc vessel density & \\
ST & 0.97 & $0.94-0.99$ & 0.048 \\
SN & 0.99 & $0.95-1.03$ & 0.579 \\
NS & 0.99 & $0.95-1.03$ & 0.587 \\
NI & 0.99 & $0.94-1.04$ & 0.736 \\
IN & 0.99 & $0.95-1.04$ & 0.715 \\
IT & 0.99 & $0.95-1.03$ & 0.566 \\
TI & 0.98 & $0.95-1.02$ & 0.450 \\
TS & $0.95-1.03$ & 0.577 \\
\hline N & 0.99 & & \\
\hline
\end{tabular}

*, adjusted with best corrected visual acuity, mean deviation, pattern standard deviation, visual field index, and $\mathrm{P}_{100}$ amplitude. pTU, peripapillary temporal upper; pST, peripapillary superior temporal; pSN, peripapillary superior nasal; pNU, peripapillary nasal upper; pNL, peripapillary nasal lower; pIN, peripapillary inferior nasal; pIT, peripapillary inferior temporal; $\mathrm{pTL}$, peripapillary temporal lower; NS, nasal superior; NI nasal inferior; IN inferior nasal; IT, inferior temporal; TI, temporal inferior; TS, temporal superior; ST, superior temporal; SN, superior nasal; OR, odds ratio; $\mathrm{Cl}$, confidence interval.

$(17.84 \% \pm 6.18 \%)$ were significantly increased when compared with normal eyes $(8.61 \% \pm 1.65 \%)(30)$. In another observational, cross-sectional case series, the perfusion defects of the $\mathrm{ONH}$ were significantly lower in NA-AION eyes compared to contralateral and control eyes (31). However, to the best of our knowledge, pVD measured by OCTA in patients with newly diagnosed NA-AION has not been studied deeply enough to highlight possible differences between cases and healthy controls.

Our study had some limitations. First, although high- quality OCTA images were included, the possible influence of ONH swelling on the detection of blood flow signal cannot be excluded. In addition, in order to exclude the potential influence of artifacts due to vascular shadowing, we did not segment the tissue into different layers, and mainly analyzed the microvascular changes at the peripapillary area using the whole-depth-mode images. Moreover, the duration of NA-AION (time elapsed from the onset of visual loss until arriving to the clinic) was self-reported by the cases, thus, we cannot identify a precise definition of the time of occurrence of the disease. Secondly, we do not know how many patients had a crowded optic disc, a known risk factor for NA-AION, because data regarding optic disc size were incomplete. Furthermore, although we included a larger sample size and more comprehensive variables than those in previous studies, the single-center, case-control study design did not allow us to reach definite conclusions, and further longitudinal studies are needed to explore vital information of diagnostic and prognostic relevance regarding vascular impairment in NA-AION.

In conclusion, decreased OCTA parameters of $\mathrm{pVD}$ were detected in the eyes of NA-AION patients. Furthermore, we observed that all the subregions of vessel density around the $\mathrm{ONH}$ were useful in the diagnosis of early NA-AION. The parameter that was the most useful for distinguishing between NA-AION and healthy eyes was the ST subregion around the optic disc. Non-invasive measurements of OCTA have better diagnostic ability to detect early-stage NA-AION, and may provide an additional biomarker for NA-AION occurrence.

\section{Acknowledgments}

Funding: This work was supported by CLINICO Meditec and Baotou Medical and Health Science Project (No. wjwkj2020100). The funding organizations had no role in the design or conduct of this research.

\section{Footnote}

Reporting Checklist: The authors have completed the STROBE reporting checklist. Available at https://dx.doi. org/10.21037/qims-21-800

Conflicts of Interest: All authors have completed the ICMJE uniform disclosure form (available at https://dx.doi. org/10.21037/qims-21-800). The authors have no conflicts of interest to declare. 
Ethical Statement: The authors are accountable for all aspects of the work in ensuring that questions related to the accuracy or integrity of any part of the work are appropriately investigated and resolved. The study was conducted in accordance with the Declaration of Helsinki (as revised in 2013). The study was approved by the Medical Ethics Committee of the Baotou Chaoju Eye Hospital and informed consent was provided by all participants.

Open Access Statement: This is an Open Access article distributed in accordance with the Creative Commons Attribution-NonCommercial-NoDerivs 4.0 International License (CC BY-NC-ND 4.0), which permits the noncommercial replication and distribution of the article with the strict proviso that no changes or edits are made and the original work is properly cited (including links to both the formal publication through the relevant DOI and the license). See: https://creativecommons.org/licenses/by-nc-nd/4.0/.

\section{References}

1. Hayreh SS. Ischemic optic neuropathy. Prog Retin Eye Res 2009;28:34-62.

2. Tournaire-Marques E. Ischemic optic neuropathies. J Fr Ophtalmol 2020;43:552-8.

3. Raizada K, Margolin E. Non-arteritic Anterior Ischemic Optic Neuropathy 2021.

4. Goldenberg-Cohen N, Kramer M, Bahar I, Monselise Y, Weinberger D. Elevated plasma levels of interleukin 8 in patients with acute anterior ischaemic optic neuropathy. $\mathrm{Br}$ J Ophthalmol 2004;88:1538-40.

5. Fang B, Liu Q, Wang J, Yu L, Liu X, Ma P, Zhao B. Evaluation of acute anterior ischaemic optic neuropathy using diffusion tensor imaging. Clin Exp Optom 2020;103:220-3.

6. Choi WJ. Imaging Motion: A Comprehensive Review of Optical Coherence Tomography Angiography. Adv Exp Med Biol 2021;1310:343-65.

7. Wu H, Sekiryu T, Sugano Y, Itagaki K, Kasai A, Shintake $\mathrm{H}$. A modified measuring method to investigate the choriocapillaris flow void of polypoidal choroidal vasculopathy with swept source optical coherence tomography angiography. Quant Imaging Med Surg 2021;11:3146-56.

8. Alam MN, Le D, Yao X. Differential artery-vein analysis in quantitative retinal imaging: a review. Quant Imaging Med Surg 2021;11:1102-19.

9. Sharma S, Ang M, Najjar RP, Sng C, Cheung CY,
Rukmini AV, Schmetterer L, Milea D. Optical coherence tomography angiography in acute non-arteritic anterior ischaemic optic neuropathy. Br J Ophthalmol 2017;101:1045-51.

10. Ghasemi Falavarjani K, Tian JJ, Akil H, Garcia GA, Sadda SR, Sadun AA. Swept-source optical coherence tomography angiography of the optic disk in optic neuropathy. Retina 2016;36 Suppl 1:S168-77.

11. Rougier MB, Delyfer MN, Korobelnik JF. OCT angiography and choroidal ischemia related to arteritic anterior ischemic optic neuropathy. J Fr Ophtalmol 2017;40:438-9.

12. Balducci N, Morara M, Veronese C, Barboni P, Casadei NL, Savini G, Parisi V, Sadun AA, Ciardella A. Optical coherence tomography angiography in acute arteritic and non-arteritic anterior ischemic optic neuropathy. Graefes Arch Clin Exp Ophthalmol 2017;255:2255-61.

13. Garway-Heath DF, Poinoosawmy D, Fitzke FW, Hitchings RA. Mapping the visual field to the optic disc in normal tension glaucoma eyes. Ophthalmology 2000;107:1809-15.

14. Obuchowska I, Mariak Z. Ischemic optic neuropathy. Pathogenesis, clinical features, diagnostics and treatment. Klin Oczna 2006;108:238-42.

15. Basile C, Addabbo G, Montanaro A. Anterior ischemic optic neuropathy and dialysis: role of hypotension and anemia. J Nephrol 2001;14:420-3.

16. Hayreh SS, Zimmerman MB. Nonarteritic anterior ischemic optic neuropathy: clinical characteristics in diabetic patients versus nondiabetic patients. Ophthalmology 2008;115:1818-25.

17. Hayreh SS, Jonas JB, Zimmerman MB. Nonarteritic anterior ischemic optic neuropathy and tobacco smoking. Ophthalmology 2007;114:804-9.

18. Reddy D, Rani PK, Jalali S, Rao HL. A study of prevalence and risk factors of diabetic retinopathy in patients with non-arteritic anterior ischemic optic neuropathy (NAAION). Semin Ophthalmol 2015;30:101-4.

19. Pantalon AD, Danielescu C, Chiseliță D. Combined etiology for bilateral and simultaneous optic neuropathy in a patient with ciancobalamin deficit and hepatitis $\mathrm{C}$ treated with peg-interferon and ribavirin. Rom J Ophthalmol 2016;60:188-94.

20. Ramunni A, Giancipoli G, Saracino A, Guerriero S, Saliani MT, Gentile MC, Sborgia C, Coratelli P. LDL-apheresis in acute anterior ischemic optic neuropathy. Int J Artif Organs 2004;27:337-41.

21. Hayreh SS, Zimmerman MB. Ocular arterial occlusive 
disorders and carotid artery disease. Ophthalmol Retina 2017;1:12-8.

22. Klintmalm G. Development of chronic renal cyclosporin-A toxicity. Nephrologie 1987;8:131-3.

23. Guo T, Zhang HR. Clinical features and carotid artery color Doppler imaging in patients with ocular ischemic syndrome. Zhonghua Yan Ke Za Zhi 2011;47:228-34.

24. Mehrabian Z, Guo Y, Miller NR, Henderson AD, Roth S, Bernstein SL. Approaches to Potentiated Neuroprotective Treatment in the Rodent Model of Ischemic Optic Neuropathy. Cells 2021;10:1440.

25. Yang Y, Zhang H, Yan Y, Gui Y, Zhu T. Comparison of optic nerve morphology in eyes with glaucoma and eyes with non-arteritic anterior ischemic optic neuropathy by Fourier domain optical coherence tomography. Exp Ther Med 2013;6:268-74.

26. Iorga RE, Moraru A, Ozturk MR, Costin D. The role of Optical Coherence Tomography in optic neuropathies. Rom J Ophthalmol 2018;62:3-14.

Cite this article as: Su Y, Zhang S, Zhang G, Liu Y, Du Z, Li D, Liu L. Quantification of peripapillary vessel density in non-arteritic anterior ischemic optic neuropathy patients with optical coherence tomography angiography. Quant Imaging Med Surg 2022;12(2):1549-1557. doi: 10.21037/qims-21-800
27. Sambhav K, Grover S, Chalam KV. The application of optical coherence tomography angiography in retinal diseases. Surv Ophthalmol 2017;62:838-66.

28. Bianchi Marzoli S, Martinelli V. Optic neuritis: differential diagnosis. Neurol Sci 2001;22 Suppl 2:S52-4.

29. Pujari A, Bhaskaran K, Sharma P, Singh P, Phuljhele S, Saxena R, Azad SV. Optical coherence tomography angiography in neuro-ophthalmology: Current clinical role and future perspectives. Surv Ophthalmol 2021;66:471-81.

30. Ling JW, Yin X, Lu QY, Chen YY, Lu PR. Optical coherence tomography angiography of optic disc perfusion in non-arteritic anterior ischemic optic neuropathy. Int J Ophthalmol 2017;10:1402-6.

31. Pierro L, Arrigo A, Aragona E, Cavalleri M, Bandello F. Vessel Density and Vessel Tortuosity Quantitative Analysis of Arteritic and Non-arteritic Anterior Ischemic Optic Neuropathies: An Optical Coherence Tomography Angiography Study. J Clin Med 2020;9:1094. 